\title{
Seudoartrosis por defecto óseo del antebrazo. A propósito de un caso.
}

Pseudoarthrosis for bone defect of the forearm. On purpose of a case.

Pavel Amigo Castañeda. ${ }^{1}$, Maylin Rodríguez Díaz. ${ }^{2}$, Rubén Ariel Olivera León. ${ }^{3}$ \& Pavel Alejandro Amigo Rodríguez. ${ }^{4}$

\begin{abstract}
.
Bone defects in the extremities are not only caused by open fractures with loss of a bone fragment, but also by tumoral or pseudotumoral lesions, osteomyelitis and congenital malformations such as pseudoarthrosis where there are several treatments within them free or nonvascularized fibula grafts with several advantages that provide a better evolution. Therefore, our objective is to show the evolution and results of a patient admitted, treated and operated on with the diagnosis
\end{abstract}

\section{Resumen.}

Los defectos óseos en las extremidades no solo se originan debido a fracturas abiertas con pérdida de un fragmento de hueso, sino también por lesiones tumorales o seudotumorales, osteomielitis y malformaciones congénitas como la seudoartrosis donde existen varios tratamientos dentro de ellos los injertos libre o no vascularizado de peroné con varias ventajas que proporcionan una mejor evolución. Por lo que nos trazamos como objetivo mostrar la evolución y los

\footnotetext{
1 Hospital Pediátrico Eliseo Noel Caamaño, Matanzas, Cuba, reynaldoamigo.mtz@infomed.sld.cu https://orcid.org/ 0000-0003-3015-9269

2 Hospital Pediátrico Eliseo Noel Caamaño, Matanzas, Cuba. maylinrd.mtz@infomed.sld.cu https://orcid.org/ 0000-0002-6600-7403

3 Hospital Pediátrico Eliseo Noel Caamaño Matanzas, Cuba. caridadcastaneda@infomed.sld.cu https://orcid.org/ 0000-0001-5623-9916

4 Universidad de Ciencias Médicas de Matanzas, Facultad de Ciencias Médicas Juan Guiteras Gener. Matanzas, Cuba, pavelamigorodriguez@gmail.com (D) https://orcid.org/ 0000-0003-3596-0637
} 
of Pseudoarthrosis due to a large bone defect of the radius which was reconstructed with an autologous free fibula graft, visualizing the course of the patient and the final result.

Keywords: Pseudoarthrosis, Bone defect, Osteosynthesis. resultados de un paciente ingresado, tratado y operado con el diagnóstico de Seudoartrosis por gran defecto óseo de la extremidad superior específicamente de radio de etiología congénita en el Hospital Provincial Pediátrico Eliseo Noel Camaño de la Provincia de Matanzas. Como resultado se muestra la evolución obtenida por el paciente al que se le aplicó tratamiento quirúrgico con el diagnóstico de Seudoartrosis por gran defecto óseo del radio al cual se le realizó reconstrucción con injerto autólogo libre de peroné, visualizándose el curso del paciente y el resultado final del mismo.

Palabras claves: Seudoartrosis, Defecto óseo, Osteosíntesis.

\section{Introducción.}

Los defectos óseos en las extremidades no solo se originan debido a fracturas abiertas con pérdida de un fragmento de hueso, sino también en lesiones tumorales o seudotumorales, osteomielitis y malformaciones congénitas como la seudoartrosis. La Seudoartrosis congénita es una enfermedad poco frecuente y rara consistente en un tipo específico de falta de unión presente desde el nacimiento asociada frecuentemente a neurofibromatosis tipo 1 en un 50-70 \%. Es provocada por una alteración segmentaria en la formación de hueso perióstico probablemente debida una función deficiente de los osteoblastos con una actividad osteoclástica aumentada pudiendo provocar fracturas espontáneas antes de los 2 años de edad. En el manejo quirúrgico existen diferentes alternativas, desde tratamientos conservadores con ortesis, injertos óseos no vascularizados, distracción ósea o injertos óseos vascularizados. Entre ellas, la resección amplia del foco de Seudoartrosis y sustitución del defecto con tejido óseo libre o vascularizado de manera precoz, ha demostrado ser la técnica más efectiva en términos de consolidación ósea. Estos últimos presentan ventajas biológicas, pero son técnicamente más demandantes. (Allende, 2017).

Este tratamiento, constituye un problema de salud para los servicios de Ortopedia y Traumatología en cualquier parte del mundo, porque alteran de forma variable las capacidades biomecánicas del hueso afectado, además de entorpecer el proceso de consolidación. (Escobedo, 2015). El período de inmovilización suele ser prolongado y los resultados funcionales poco satisfactorios. El fracaso de la consolidación causa invalidez, tiene efectos socio-económicos considerables y están fundamentalmente asociados con la 
pérdida del trabajo y el incremento del costo del tratamiento Por estas razones, los grandes defectos óseos requieren tratamientos específicos. El relleno de los daños en el hueso con algún tipo de injerto permite acortar el tiempo de consolidación y mejora el pronóstico funcional. Por lo que el objetivo común ha sido desde hace algún tiempo acortar el tiempo de consolidación con procederes como las Transportaciones óseas, los Injertos óseos libres y los Injertos óseos vascularizados. (Allende, 2017, Escobedo, 2015).

Haciendo un poco de Historia se le atribuye a Van Meekren en el 1668 haber sido el pionero en implantar hueso heterólogo con el fin de reparar defecto óseo en el cráneo. En 1809 se reporta primer trasplante injerto óseo antólogo y no es hasta 1879 Mac Ewen informa el uso de injerto autólogo para tratar defectos óseos. En el 1960 Mac Keever notifica el uso de hueso autólogo de cresta iliaca para reparar defectos óseos de tibia y en los 1974 trabajos experimentales de Ostrup y Fredrickson muestran la utilización ya de injertos vascularizados de peroné. Posteriormente en el 1975 L. Taylor pone a punto la técnica quirúrgica utilizando injerto vascularizado de peroné con un abordaje posterior para la toma del injerto. Ya en el 1979 Gilbert describe la vía de abordaje lateral simplificando la técnica y acortando el tiempo quirúrgico. Estos injertos pueden tomarse de 2 maneras, una de forma directa del propio paciente o mediante tejido liofilizado procedente de banco de hueso. (Delgado, 2019). A pesar que el injerto vascularizado es el proceder ideal para suplir defectos de más de $6 \mathrm{~cm}$ es una técnica de microcirugía muy laboriosa y compleja que requiere personal entrenado además de un instrumental especifico y ambiente hospitalario de alta complejidad. Cada año en los Estados Unidos, se utilizan cerca de 450 000 injertos óseos de diversos tipos, en afecciones relacionadas con defectos del tejido óseo. (Vejarano, 2015).

La utilización de injerto óseo es frecuente en las cirugías traumatológicas, siendo necesario en ocasiones utilizar grandes cantidades de aporte óseo tanto para defectos como en pacientes con pseudoartrosis. Son escasos los estudios en Latinoamérica que describan los resultados obtenidos con estas nuevas y prometedoras técnicas ortopédicas. La utilización del autoinjerto es de fácil acceso e incluso, es el más económico, y el que tiene las propiedades de osteogénesis, osteoconducción y osteoinducción. Sin embargo, está asociado con un alto grado de morbilidad con una limitación en la cantidad por extraer, y con la presencia de complicaciones, como dolor, lesión vasculo-nerviosa o infección. La utilización de aloinjerto es cada vez más frecuente en la práctica ortopédica, ya que tienen diferentes capacidades para activar la formación ósea. (Arias, 2018).

Características de peroné y ventajas del mismo para su selección como hueso donante.

El peroné constituye una varilla larga, biológica y fuerte, la cual posee 3 corticales gruesas en un corte transversal que lo hace resistente y versátil para reconstrucción de defectos en huesos largos. La extremidad proximal posee un cartílago articular muy útil en la reconstrucción de superficies articulares como la del hombro y la de la muñeca. En cuanto a su vascularización es irrigado por la arteria peronea rama del tronco tibio peroneo, corriendo paralelo al peroné a lo largo de toda la pierna. Emite rama para la vascularización endostica como lo es la arteria nutricia unión tercio proximal y medio y 
numerosas arterias perióticas que irrigan todo su recorrido anastomosándose entre sí. El drenaje se realiza por venas que corren paralelas a los vasos arteriales. La cabeza del peroné se irriga por ese mismo sistema en adultos, aunque en el niño es importante tener en cuenta una rama de la arteria tibial anterior denominada arteria del cuello del peroné y arteria genicular inferior que se deben tener bien presente para mantener la viabilidad del cartílago de crecimiento. La cara externa de la piel de la pierna se irriga por 2 o 3 ramas voluminosas que pasan entre los músculos peroneos y a través del sóleo por lo que si se va a obtener un injerto osteocutaneo debe incorporarse una pequeña porción del soleo para proteger estos vasos. (Ferrer, 2008). Por todo esto nos trazamos como objetivo mostrar la evolución y los resultados de un paciente ingresado, tratado y operado con el diagnóstico de Pseudoartrosis por gran defecto óseo de la extremidad superior específicamente de radio.

\section{Metodologia.}

Se realiza la presentación de un caso interesante, donde se muestran los resultados obtenidos mediante el estudio y tratamiento aplicado de dicho paciente, con diagnóstico de Pseudoartrosis por gran defecto óseo de la extremidad superior específicamente de radio de etiología congénita en el Servicio de Ortopedia y Traumatología del Hospital Provincial Pediátrico Eliseo Noel Caamaño de la provincia de Matanzas, durante el año 2019.

\section{Resultados.}

Presentación del caso. Paciente: masculino de 11 años de edad, de color mestizo de la piel. Con un desarrollo estaturoponderal retardado según evaluación de especialista Endocrinología pediátrica, teniendo en cuenta entre otros aspectos la escala de peso y talla. Procedencia rural, cuya labor que realiza estudiante del nivel primario de educación. En cuanto a los antecedentes, paciente que según su mamá sufre caída de sus pies y recibe trauma a nivel de antebrazo izquierdo por lo que comienza con dolor e imposibilidad de realizar funciones, por lo que se interpreta, según el Rx y el cuadro clínico, como una fractura diafisaria de cúbito y radio por lo que se trató inicialmente con intramedular liso no bloqueado para radio y cúbito por encontrarse los fragmento óseos con determinado grado de desplazamiento y que no mostró signos de consolidación a los cuatro meses de operado, perdiendo la fijación por migración de los alambres.

Es evaluado por el servicio provincial de cirugía ortopédica pediátrica e interpretada una como una Seudoartrosis no viable de cúbito y radio por el exámen físico y las imágenes radiológicas. Por lo que se decide reintervenir y se coloca aparato de osteosíntesis extrafocal en radio donde se realiza resección del foco Seudoartrosico y se decide por el defecto presentado realizar transportación ósea de proximal a distal.

En el curso de la evolución clínica y radiológica una vez concluida el proceso de transportación no se percibe consolidación clínica ni radiológica y ante el inminente fallo del proceder por rechazo a la osteosíntesis y sepsis de los orificios de los alambres, se discute en colectivo nuevamente y se procede a interrogar a la familia donde se precisan antecedentes de familiares con Seudoartrosis congénitas en tibia asociado a 
Neurofibromatosis, por lo que se decide 2 meses después de no existir signos de sepsis activa según la clínica y los complementarios pertinentes realizar resección del foco Seudoartrosico dejando un defecto de alrededor de $6 \mathrm{~cm}$ y colocar injerto óseo autólogo libre en bloque del peroné y fijándolo con intramedular y fijador externa monopolar modelo profesor Rodrigo Álvarez Cambras. Presentando una evolución satisfactoria cumpliéndose nuestro objetivo.

Fig. 1. Imagen radiológica donde se visualiza el defecto de radio de más de $6 \mathrm{~cm}$.

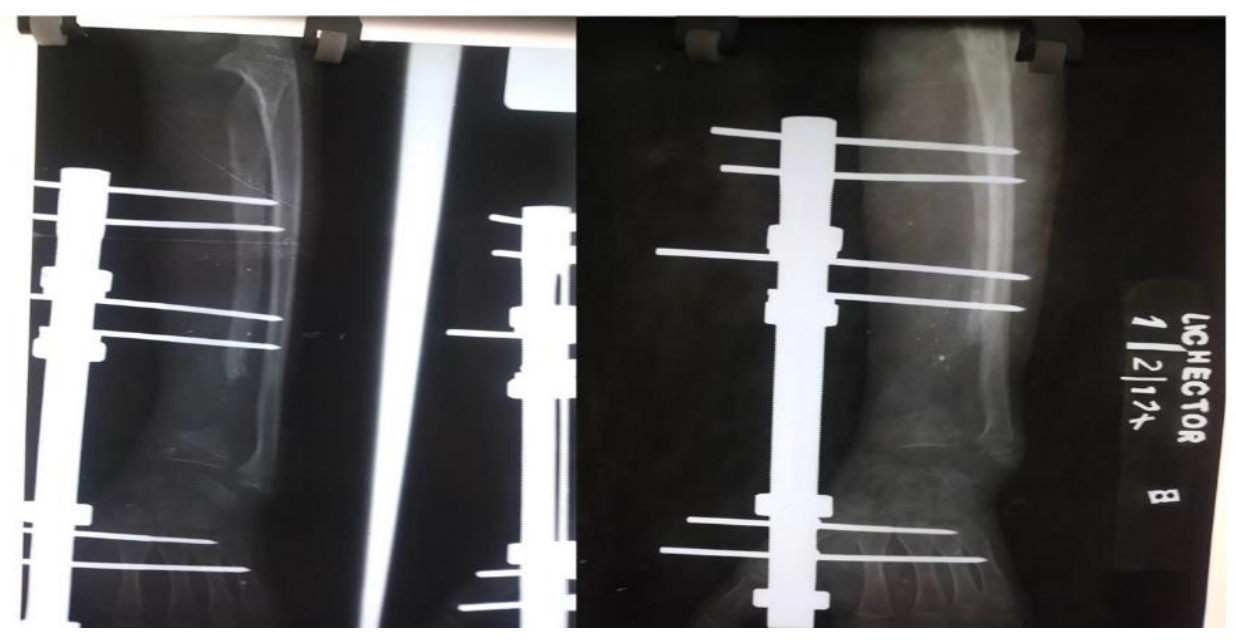

Fuente: Imagen tomada por los autores.

Fig. 2. Imagen del transoperatorio donde se toma la muestra del injerto libre de peroné.

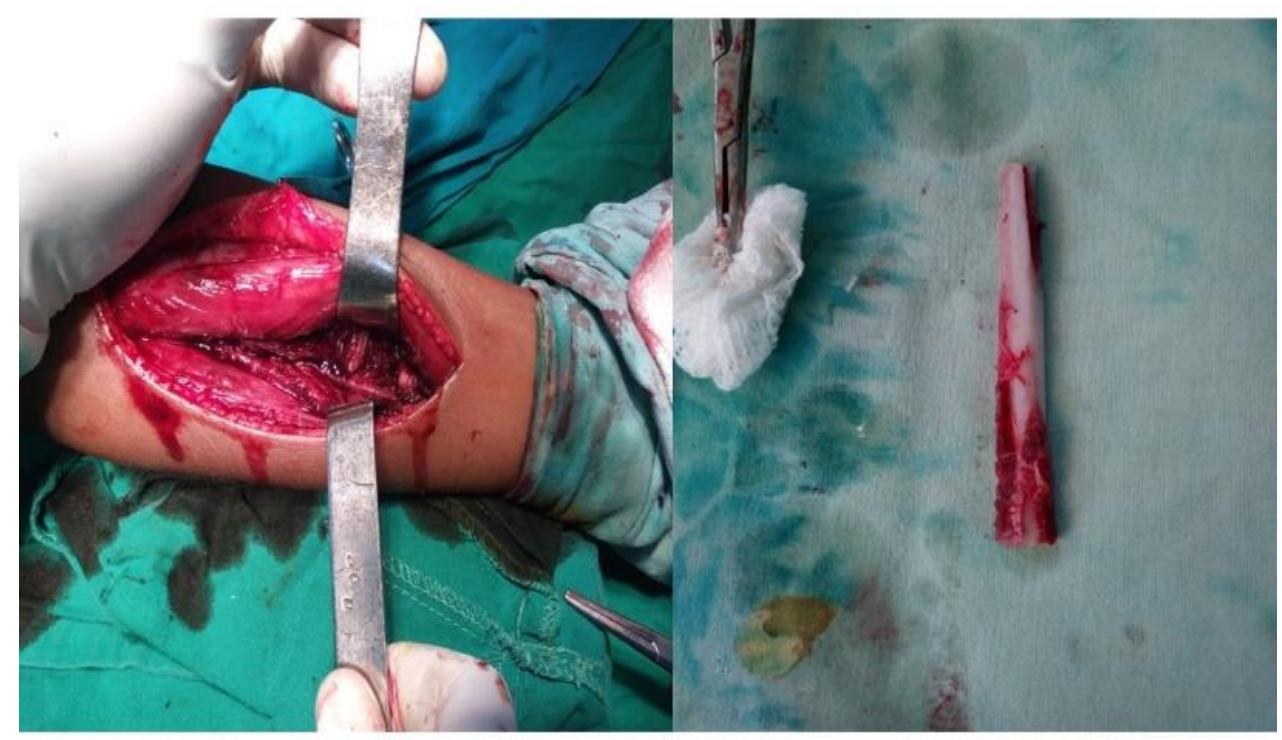

Fuente: Imagen tomada por los autores. 
Fig. 3. Imágenes después de colocar el injerto libre de peroné y la fijación del mismo.

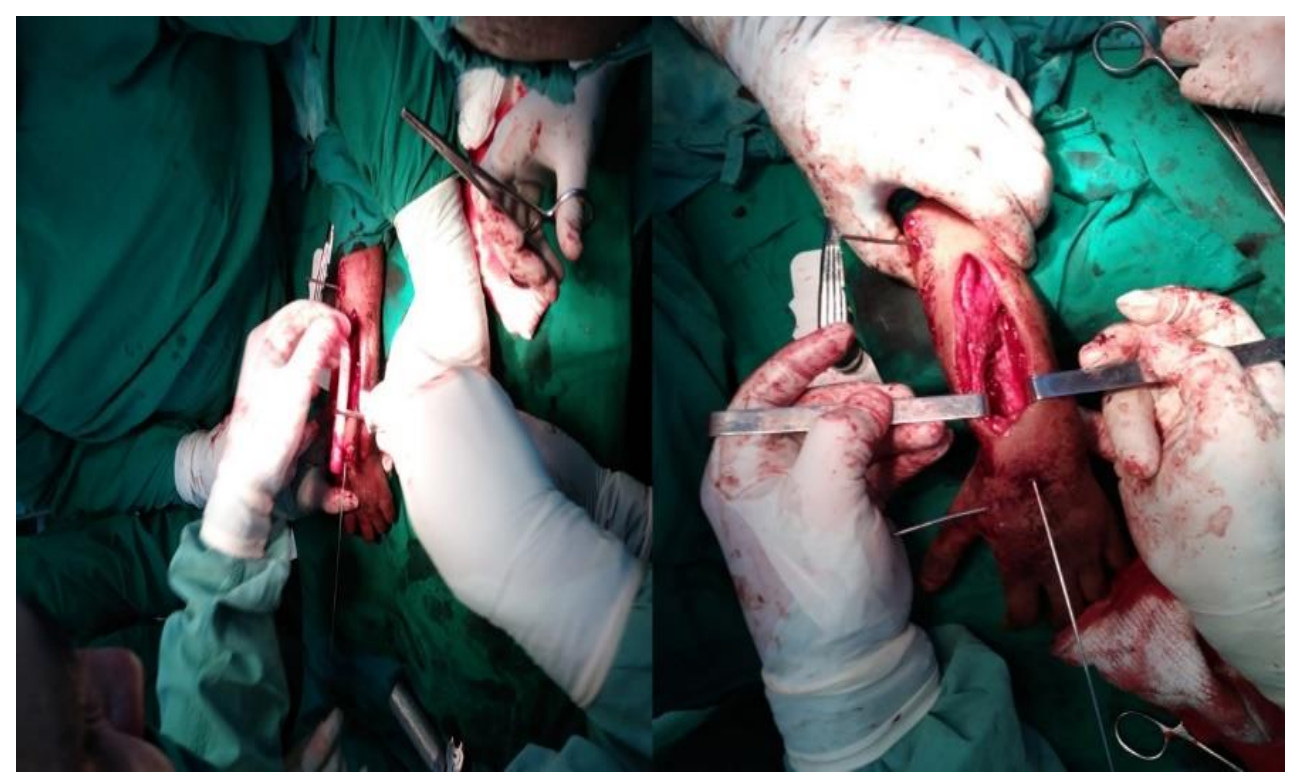

Fuente: Imágenes tomadas por los autores.

Fig. 4. Imagen radiológica después de colocar el injerto libre de peroné y su osteosíntesis.

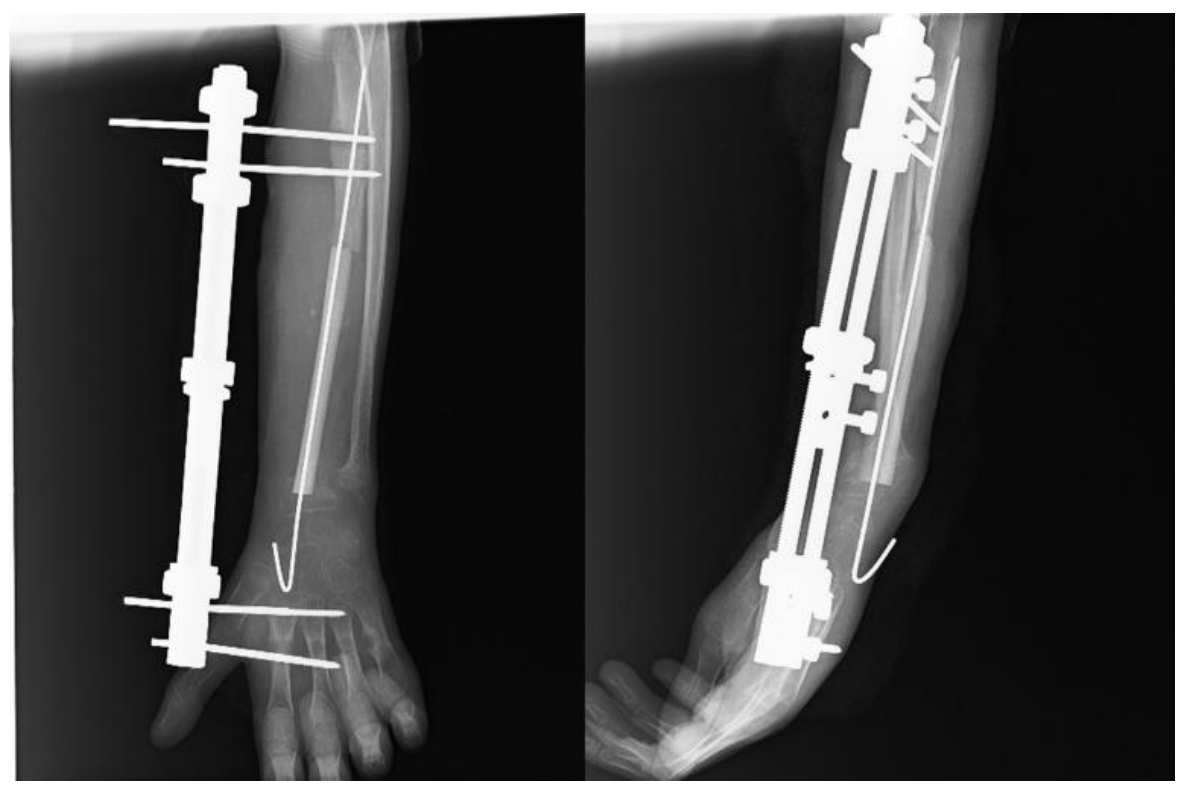

Fuente: Imágenes tomadas por los autores.

Durante el periodo en sala no presentó complicación alguna y fue dado de alta a los 20 días donde se combinaron tratamientos de campos magnéticos y ozonoterapia local en bolsa específicamente.

Se le realizaron radiografías a $\operatorname{los} 3,6,12,16$ semanas retirando el intramedular inicialmente para que sirviera de estímulo a la circulación endostica, cumpliendo el 
principio de recanalización y a las 20 semanas se decide retira el aparato de osteosíntesis extrafocal monopolar Ralca.

Para evaluar el proceso de consolidación ósea desde el punto de vista radiológico decidimos evaluarlo según la escala de evaluación de la consolidación de Montoya donde evalúa la presencia y visualización del trazo de la fractura.

Escala de Evaluación de la Consolidación. Montoya.

Estadio I Reacción periótica sin callo.

Estadio II Callo con trazo de fractura o de unión visible.

Estadio III Callo con trazo de fractura o de unión visible parcialmente.

Estadio IV Desaparición del trazo de fractura o de unión.

Los implantes fueron retirados cuando tanto proximal y como distalmente el injerto se encontraba en Estadio IV. (Ferrer, 2008).

Fig. 5. Imagen radiológica de consolidación del injerto libre colocado y la zona donadora.

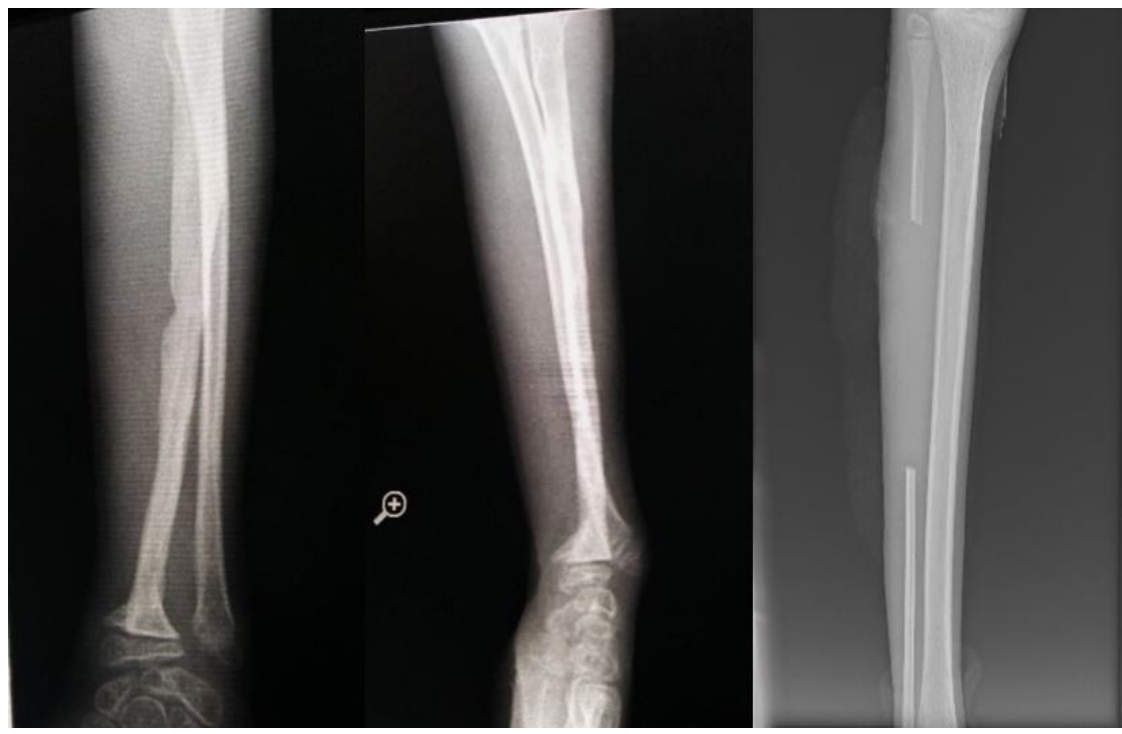

Fuente: Imágenes tomadas por los autores.

El defecto óseo en la cirugía ortopédica y traumatológica continúa representando un problema difícil de tratar para los ortopédicos, ya que implica un sostenido aumento en hospitalización, riesgo de complicaciones y la elevación de los gastos. (Sallés, 2001, Pancorbo, 2016, Wiese, 2010). La seudoartrosis congénita es una enfermedad rara consistente en un tipo específico de falta de unión presente desde el nacimiento asociada frecuentemente a neurofibromatosis tipo 1. En el manejo quirúrgico es la resección amplia del foco de Pseudoartrosis y sustitución del defecto con tejido óseo libre o vascularizado que, realizado de manera precoz, ha demostrado ser la técnica más efectiva en términos de consolidación ósea. El injerto libre vascularizado de peroné combina la viabilidad del 
injerto esponjoso con la estabilidad del cortical, proporcionando un soporte óseo fuerte y vivo para las grandes pérdidas de sustancia ósea y ricamente vascularizado, lo que lo hace resistente y útil en zonas pobremente irrigadas o previamente infectadas. Tiene como desventaja que requiere para su realización un equipo de cirujanos entrenados y un ambiente hospitalario de alta complejidad, nivel alto de consolidación, bajo número de complicaciones y morbilidad prácticamente nula en la zona dadora. (Arias, 2018).

Los colgajos microquirúrgicos permiten disponer una variedad de tejidos que posibilitan la reconstrucción de defectos complejos secundarios a exéresis oncológica, traumatismos severos, infecciones y malformaciones congénitas o del desarrollo. El colgajo de peroné es un colgajo libre microvascularizado que permite aportar hueso, piel y músculo, y está indicado para la reconstrucción del esqueleto apendicular y mandibular. (Wiese, 2010). El colgajo de peroné aporta un hueso largo y fuerte y puede incluirse un componente fasciocutáneo, muscular e isla de piel adyacente. La primera circunstancia a considerar es la necesidad de tejidos para reconstruir un defecto concreto y la importancia relativa de cada uno de los componentes: hueso, cobertura externa, cobertura interna o músculo.

Estudios recientes respecto del injerto libre de peroné han sido enfocados en la alta morbilidad de la zona dadora. La prevalencia del dolor de tobillo ha sido reportada entre el 10 al $40 \%$, pero su etiología no es clara. La literatura es vaga acerca del tamaño del peroné distal necesario para mantener la estabilidad. Algunos autores identificaron causas subjetivas de dolor en el tobillo, otros identificaron inestabilidades groseras incluso con necesidad de estabilización quirúrgica, y otros reportaron la combinación de ambas. (Wiese, 2010). Estudios clínicos y biomecánicos determinaron la importancia de la longitud del peroné distal remanente, pero ninguno cuantificó la longitud necesaria para mantener la estabilidad. El peroné es un hueso resistente, con longitud suficiente para la reconstrucción de grandes defectos. Su uso para reemplazar los defectos óseos de los huesos largos, depende de la calidad vascular de los tejidos vecinos, las células osteogénicas sobrevivientes del injerto para la supervivencia y de la formación del tejido óseo. (Sallés, 2001). Puede obtenerse por toma directa del propio paciente o mediante tejido liofilizado procedente del Banco de Hueso. Desde finales del siglo pasado y durante los primeros años del presente, un importante grupo de autores (Masquelet, 2010, Litchte, 2011) han desarrollado un gran número de trabajos investigativos, que hoy día se encuentran en fase de aplicación, como son los biomateriales, los diversos factores de crecimiento, la importancia de la angiogénesis y de las proteínas en el proceso de curación ósea. (Cannada, 2010, Hankenson, 2011, Le, 2001). Todas estas investigaciones han permitido desarrollar múltiples tecnologías de osteosíntesis, con vistas a afectar lo menos posible el área de unión de los segmentos óseos. No obstante, se ha demostrado que a pesar de lo óptimo de tecnologías mínimo invasivas, es de fundamental aplicación en las áreas de defecto, injertos óseos, células madres, estimulación eléctrica, biomateriales con propiedades diversas (osteoconductotes, osteoinductores, osteogénicos), que a través de diferentes mecanismos permitan un crecimiento óseo adecuado y la curación ósea. (Hankenson, 2011, Le, 2001). 
Muchos autores consideran importante valorar en toda osteosíntesis la necesidad del empleo de injerto o biomaterial, para permitir el efecto de andamiaje tan necesario para logar el crecimiento óseo, ayudado o no por otros mecanismos, como la aplicación de las células madres con vistas a lograr la curación ósea, coincidiendo con diversos autores. (Masquelet, 2010, Litchte, 2011) que es de vital importancia evaluar a cada caso de forma individual como una norma. El injerto vascularizado de peroné es considerado, por muchos, la técnica de elección en el tratamiento de defectos en las extremidades que sobrepasan los seis centímetros, a pesar de ser una técnica microquirúrgica muy compleja y laboriosa. Algunos autores plantean que los injertos óseos no vascularizados, representan una importante alternativa de tratamiento en defectos menores de seis centímetros, con buena viabilidad de los tejidos blandos circundantes. Este tipo de injerto presenta una lenta e incompleta neovascularización debido a la mezcla de hueso necrótico y viable, lo que disminuye su resistencia mecánica. (Wiese, 2010).

El uso de injerto libre de peroné en pacientes estudiados, ha tenido principalmente dos objetivos: favorecer la ontogénesis y servir de soporte mecánico. Las fases de cicatrización del injerto y formación ósea ocurren a través de tres vías (Arias, 2018): a) Osteogénesis, donde ocurre la síntesis de hueso nuevo a partir de células derivadas del injerto o del huésped; b) Osteoinducción, se inicia por medio de la transformación de células mesenquimales indiferenciadas perivasculares de la zona receptora, a células osteoformadoras en presencia de moléculas reguladoras del metabolismo óseo. Ello produce una invasión de vasos sanguíneos y de tejido conectivo, al injerto óseo proveniente del hueso huésped; y c) Osteoconducción, donde ocurre un crecimiento tridimensional de tejido perivascular y células madres mesenquimatosas, desde la zona receptora del huésped, hacia el injerto que realiza la función de esqueleto.

La cortical sola como injerto, provee una estructura muy resistente. Para su cicatrización, solo se da la fase de osteoconducción. Puede actuar como barrera para la invasión del tejido blando, comportándose de manera similar a una membrana microporosa usada para la regeneración ósea guiada (Delgado, 2019). Algunos autores con la técnica empleada en este estudio, tiene un porcentaje de resolución en los pacientes entre el 61 y $81 \%$ y notifican también buenos resultados morfológicos y funcionales en esqueletos inmaduros. Otros, reportan con el uso de este método un $40 \%$ de reabsorción del injerto, y tiempos de consolidación entre 28 y 58 semanas, con un $50 \%$ de incidencia de refractura. (Hankenson, 2011).

Minami y colaboradores plantean, que a mayor longitud del defecto, se puede incrementar el índice de fracturas por fatiga y mala alineación. (Minami, 2000) De Gauzy y Accadbled encuentran varias refracturas del injerto de peroné en la porción proximal del fémur, comenzando desde las doce semanas por el inicio de la carga de peso. La pérdida de la alineación en un caso tuvo que ver más con la estabilidad quirúrgica que con la migración del injerto. (De Gauzy, 2009). Delgado y colegas fueron los primeros en describir en 1975, el uso de peroné vascularizado en fracturas tibiales con grandes defectos e irrigación deficiente de los tejidos circundantes. (Delgado, 2019). Sallés y colaboradores por su parte, 
utilizando injerto de peroné vascularizado, encuentran en la evaluación posoperatoria que el $80 \%$ de sus pacientes consolidó en menos de 30 semanas. El injerto aplicado por este autor, a diferencia del injerto libre, tiene la ventaja de conservar la irrigación propia y no depender de los tejidos vecinos para su nutrición, por lo que se comporta como una fractura segmentaria, lo que justifica el menor tiempo de consolidación. (Sallés, 2001).

Yera y colaboradores en un estudio con pacientes con defectos óseos, ocasionados por lesiones infecciosas de las extremidades, consideran que la transportación ósea fue mucho más útil que el injerto óseo de peroné ya que con la transportación se logra recuperar el espacio óseo perdido, llevando hueso vascularizado de una zona cercana, sin tener que tomarlo de otra zona del mismo paciente, aunque reconoce que en pacientes con infecciones óseas, muchos cirujanos emplean el injerto óseo por hacer más fácil y rápida la evolución. (Yera, 2004).

El injerto de hueso antólogo o autoinjerto, ha sido el material clásicamente elegido por cirujanos ortopedistas para el tratamiento de defectos óseos. Es considerado el "patrón de oro, sin embargo, las desventajas relacionadas con la morbilidad de la zona dadora (habitualmente cresta ilíaca), tales como dolor post operatorio, sangrado, riesgo de infección, cantidad limitada de tejido óseo y necesidad de sacrificar estructuras normales, restringen su aplicación. Las alternativas disponibles son el injerto óseo obtenido de otro paciente (aloinjertos) Las ventajas de los aloinjertos sobre los autoinjertos están en relación a su más fácil disponibilidad, ausencia de morbilidad de la zona dadora, menor tiempo quirúrgico y menor sangrado, capacidad de reconstrucción grandes defectos, etc. Entre sus desventajas están los tiempos de incorporación más prolongados y la capacidad osteoconductiva y osteoinductoras menores comparada con el autoinjerto. El punto más conflictivo está en relación con la transmisión de enfermedades. Afortunadamente, la adecuada selección de donante, el desarrollo de las técnicas de estudio microbiológico, preservación de tejidos y esterilización con distintas dosis de radiación gamma han reducido esta complicación. (Bornot, 2017, Estefan, 2017).

Los defectos óseos $>6 \mathrm{~cm}$ en los huesos largos plantean un problema difícil de solucionar en la reconstrucción del miembro superior. El peroné vascularizado se ha convertido en el principal método de reconstrucción por sus ventajas biológicas en lugares donde se pueda realizar. (Estefan, 2017)

\section{Conclusiones.}

- El injerto libre de peroné continúa siendo una técnica efectiva para resolver grandes defectos óseos en aquellos servicios que no cuenten con Banco de Hueso para obtener hueso liofilizado, ni posibilidad de realizar injertos vascularizados.

\section{Referencias bibliográficas.}

Allende V., Masquijo J. (2017); Seudoartrosis congénita de antebrazo asociada a luxació De Radio proximal: tratamiento quirúrgico mediante antebrazo de un 
solo hueso. Rev Asoc Argent Ortop Traumatol (2017); 82 (3): 242-248 ISSN 1852-7434 (en línea) ISSN 1515-1786 (impresa) http://dx.doi.org/10.15417/420

Arias, R M., Cortez, W X., Novillo, E.E. Et al (2018). Uso de aloinjerto esponjoso en Fracturas y pseudoartrosis en defectos óseos: experiencia en el Hospital LuisVernaza. Archivos Venezolanos de Farmacología y Terapéutica, 37(4), 396399.

Bornot Duránd Y., Ferrer Matos G., Font Ávila M E, (2017). Pseudoartrosis no viable de tercio distal del cúbito. Presentación de un caso. Correo Científico Medico de Holguín ISSN 1560-4381 CCM;

Cannada LK. (2010) Viable Bone and circulatory factors for survival of bone grafts. OrthopClin North Am [Internet]. [Citado 4 Feb 2015]; 41(1): 5-13. Disponible en: http://linkinghub.elsevier.com/retrieve/pii/S0030589809000613?via=sd

Delgado del Caño, J., García López, R., Rodríguez de Oya, (2019). Pseudoartrosis séptica. Actualización en manejo y tratamiento Unidad de Traumatología. Hospital Monográfico Asepeyo Coslada. Madrid. Revista Española de Traumatología Laboral Vol. 2. Fasc. 1. Núm. 3. Mayo ISSN: 26050579 (impreso) 2659-7535 (online)

De Gauzy J., Accadbled F., (2009). Case Report: Histologic Study of a Human Epiphyseal. Transplant at 3 Years after Implantation. Clin Orthop Relat Res.; 467(7):1915-20.

Escobedo-Troncoso, VM., Cantú-Morales, D., (2015) Formación de un solo hueso antebrazo, en pseudoartrosis infectada de cúbito proximal. Acta ortop. Mex [online]. vol.29, n.2 [citado 2020-06-13], pp.114-117. Disponible en: http://www.scielo.org.mx/scielo.php?script=sci_arttext\&pid=S2306

Estefan M., Díaz Dilernia F., Gallucci G. Et al. (2017) Experiencia inicial en un Centro de Alta Complejidad con el injerto vascularizado de peroné en defectos óseos Segmentarios del miembro superior. Rev Asoc Argent Ortop Traumatol; 82 3): 170-181. ISSN 1852-7434 (en línea) ISSN 1515-1786 (impresa) http://dx.doi.org/10.15417/598

Ferrer Y., Vergara-Pagés J., (2008); Injertos en cirugía ortopédica. Rev Portalesmédicos.com. 3(6):1026.

Hankenson KD., Dishowitz M., Gray C., Et al. (2011) Angiogenesis in bone regeneration. Injury [Internet]. [Citado $4 \mathrm{Feb} 2015$ ]; 42(6). Disponible en: http://www.sciencedirect.com/science/article/pii/S002013831100129X

Le AX, Miclau T, Hu D, A., Et al. (2001) Molecular aspects of healing in stabilized and non-stabilized fractures. J Orthop Res [Internet]. [Citado 4 Feb 2015]; 19(1):78- 
84. Disponible en: http://onlinelibrary.wiley.com/doi/10.1016/S07360266\%2800\%2900006-1/abstract

Litchte P., Pape HC., Pufe T., Et al. (2011). Scaffolds for bone healing: Concepts, Materials and evidence. Injury [Internet] [Citado 4 Feb 2015]; 42:569-73. Disponible en http://www.researchgate.net/profile/Hans_Pape/publication/51047970

Masquelet AC., Begue T., (2010). The concept of induced membrane for reconstruction of Long bone defects. Orthop Clin N Am [Internet]. [Citado 4 Feb 2015]; 41(1):27-37 Disponible en: http://www.sciencedirect.com/science/article/pii/S0030589809000716

Minami A., Kasashima T., Iwasaki N., Et al. (2000). Vascularized fibular grafts an experience of 102 patients. J Bone Joint Surg.; 82B (7):1022-4

Pancorbo Sandoval E., cols. (2016). Tratamiento de las fracturas complejas diafisarias de tibia con hidroxiapatita coralina porosa HAP-200. Revista Electrónica Matanzas. ISSN 1684-1824. Vol. 38 No 3 mayo-junio. Disponible en (http://www.revmedicaelectronica.sld.cu/index.php/rme)

Sallés Betancourt G., Cabrera Viltres N., Álvarez L., Et al. (2001); Injerto vascularizado de Peroné y minifijador externo en defectos óseos de miembro superior. Rev cubana Ortop Traumatol.; 15 (1-2):32-4.

Vejarano-Solano, JC., Ruiz-Semba, CF., Ganoza-Arróspide, CJ. Et al (2015). Reconstrucción de defectos óseos segmentarios postraumáticos mediante técnica de inducción de Membrana Rev Med Hered. 26:76-85.

Wiese A., Pape HC., (2010) Bone defects caused by high-energy, bone loss, infected Nonunions and Nonunions. Orthop Clin N Am [Internet]. [Citado 4 Feb 2015]; 41(1). http://www.sciencedirect.com/science/article/pii/S0030589809000662

Yera J., Marrero L., Garrido A., Et al. (2004); Cirugía de salvataje en las grandes lesiones 18(2). [Citado infectadas de las extremidades. Rev cubana Ortop Traumatol 2012-02- 03], pp. 0-0. Disponible en: http://scielo.sld.cu

\section{Ciencia}


PARA CITAR EL ARTÍCULO INDEXADO.

Amigo Castañeda, P., Rodríguez Díaz, M., Olivera León, R. A., \& Amigo Rodríguez, P. A. (2021). Seudoartrosis por defecto óseo del antebrazo. A propósito de un caso . Anatomía Digital, 4(2), 17-29. https://doi.org/10.33262/anatomiadigital.v4i2.1614

\section{¿Ciencia}

El artículo que se publica es de exclusiva responsabilidad de los autores y no necesariamente reflejan el pensamiento de la Revista Anatomía Digital.

El artículo queda en propiedad de la revista y, por tanto, su publicación parcial y/o total en otro medio tiene que ser autorizado por el director de la Revista Anatomía Digital.
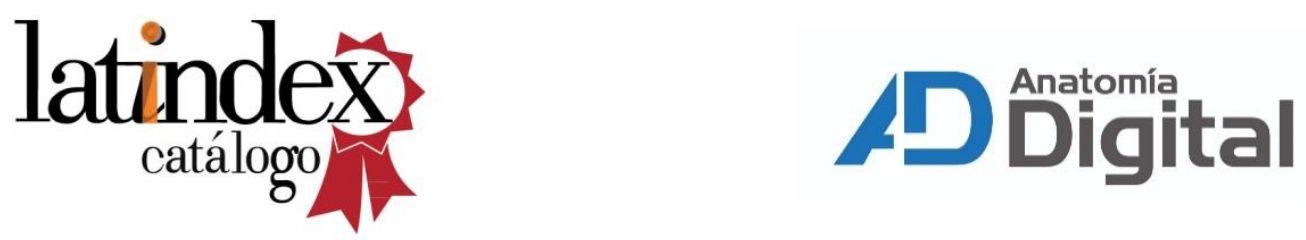